\title{
A small molecule inhibitor of atypical protein kinase $C$ signaling inhibits pancreatic cancer cell transformed growth and invasion
}

\author{
Amanda M. Butler ${ }^{1}$, Michele L. Scotti Buzhardt ${ }^{2}$, Eda Erdogan ${ }^{3}$, Shuhua Li ${ }^{1}$, Kristin \\ S. Inman ${ }^{1}$, Alan P. Fields ${ }^{1}$ and Nicole R. Murray ${ }^{1}$ \\ ${ }^{1}$ Department of Cancer Biology, Mayo Clinic, Jacksonville, FL, USA \\ ${ }^{2}$ Genoptix/Novartis Molecular Diagnostics, Carlsbad, CA, USA \\ ${ }^{3}$ Department of Biomedical Sciences and Pathobiology, Virginia Polytechnic Institute and State University, Blacksburg, VA, \\ USA \\ Correspondence to: Nicole R. Murray, email: murray.nicole@mayo.edu \\ Keywords: atypical PKCs, aurothiomalate, pancreatic cancer, transformed growth, invasion \\ Received: July 30, $2014 \quad$ Accepted: March 10, $2015 \quad$ Published: April 14, 2015
}

This is an open-access article distributed under the terms of the Creative Commons Attribution License, which permits unrestricted use, distribution, and reproduction in any medium, provided the original author and source are credited.

\section{ABSTRACT}

Pancreatic cancer is highly resistant to current chemotherapies. Identification of the critical signaling pathways that mediate pancreatic cancer transformed growth is necessary for the development of more effective therapeutic treatments. Recently, we demonstrated that protein kinase $\mathrm{C}$ iota $\left(\mathrm{PKC}_{\mathrm{l}}\right)$ and zeta (PKC $)$ promote pancreatic cancer transformed growth and invasion, by activating Rac1 $\rightarrow$ ERK and STAT3 signaling pathways, respectively. However, a key question is whether $\mathrm{PKC}_{1}$ and $\mathrm{PKC \zeta}$ play redundant (or non-redundant) roles in pancreatic cancer cell transformed growth. Here we describe the novel observations that 1) $\mathrm{PKC}_{1}$ and $\mathrm{PKC}$ are non-redundant in the context of the transformed growth of pancreatic cancer cells; 2) a gold-containing small molecule known to disrupt the PKC1/Par6 interaction, aurothiomalate, also disrupts PKCZ/Par6 interaction; 3) aurothiomalate inhibits downstream signaling of both $\mathrm{PKC}_{1}$ and $\mathrm{PKC}_{3}$, and blocks transformed growth of pancreatic cancer cells in vitro; and 4) aurothiomalate inhibits pancreatic cancer tumor growth and metastasis in vivo. Taken together, these data provide convincing evidence that an inhibitor of atypical PKC signaling inhibits two key oncogenic signaling pathways, driven non-redundantly by $\mathrm{PKC}_{1}$ and $\mathrm{PKC}$, to significantly reduce tumor growth and metastasis. Our results demonstrate that inhibition of atypical PKC signaling is a promising therapeutic strategy to treat pancreatic cancer.

\section{INTRODUCTION}

Pancreatic cancer remains the fourth leading cause of cancer deaths in the United States [1]. Pancreatic cancer has a high propensity for resistance to current chemotherapy treatments; thus molecular targeting of multiple pathways necessary for tumor cell growth and maintenance may be required for successful therapeutic intervention. Recently, we demonstrated a role for the atypical protein kinase $\mathrm{C}$ (aPKC) isozymes, $\mathrm{PKCl}$ and $\mathrm{PKC} \zeta$, in the transformed growth and invasive phenotype of pancreatic cancer cells $[2,3]$. Aurothiomalate (ATM) forms a thio-gold adduct with a cysteine residue within the Phox-Bem1 (PB1) domain of $\mathrm{PKCl}$ (Cys69), thereby blocking PB1 domain-mediated interactions with components of $\mathrm{PKCl}$ 's pro-cancer signaling complex, and inhibiting $\mathrm{PKCl}$ oncogenic function [4]. Similar to genetic inhibition of $\mathrm{PKCl}$, ATM inhibits soft agar colony formation of lung cancer cells in vitro and orthotopic lung tumor growth and proliferation in vivo [4-6]. Auranofin (ANF), a second small molecule gold-containing compound in the same chemical class as ATM, inhibits $\mathrm{PKCl}$ signaling in ovarian cancer, via a mechanism of action similar to that of ATM [7]. PKC $\zeta$ is structurally similar to $\mathrm{PKCl}$ and also contains a cysteine residue in the protein-binding region of the PB1 domain (Cys68); therefore these gold-containing compounds are predicted to disrupt $\mathrm{PKC} \zeta$-mediated downstream signaling.

In the present study, we show that $\mathrm{PKC} 1$ and $\mathrm{PKC} \zeta$ play non-redundant, required roles in pancreatic cancer 
cell transformed growth. We demonstrate for the first time that ATM disrupts binding of $\mathrm{PKC} \zeta$ to partitioning defective 6 homolog (Par6), an aPKC signaling partner, and that inhibition of expression of Par6 significantly reduces transformed growth and invasion of pancreatic cancer cells, suggesting that aPKC/Par6 signaling is an important mediator of the transformed phenotype of pancreatic cancer cells. Furthermore, ATM inhibits the transformed growth of pancreatic cancer cell in vitro and tumor formation in vivo, demonstrating the therapeutic potential of disrupting aPKC signaling.

\section{RESULTS}

\section{$P K C \imath$ and $P K C \zeta$ play non-redundant roles in the transformed phenotype of pancreatic cancer}

We have previously shown that inhibition of expression of either $\mathrm{PKCl}$ or $\mathrm{PKC} \zeta$ significantly reduces pancreatic cancer cell anchorage-independent growth and cellular invasion [2, 3]. Since $\mathrm{PKCl}$ and

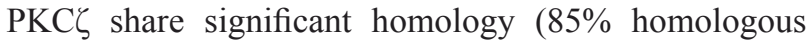
in the catalytic domains [15]), one interpretation of this observation is that the atypical PKC isozymes play redundant roles in the transformed growth of pancreatic cancer cells. We therefore evaluated the ability of $\mathrm{PKCl}$ to reconstitute the effects of $\mathrm{PKC} \zeta$ knockdown, and $\mathrm{PKC} \zeta$ to reconstitute the effects of $\mathrm{PKCl}$ knockdown, on the transformed phenotype of pancreatic cancer cell lines. Panc-1 cells expressing control NT RNAi, PKCi RNAi or PKC $\zeta$ RNAi were co-transfected with empty (control) vector, or vector expressing $\mathrm{PKCl}$ or $\mathrm{PKC} \zeta$ (Figure 1A). As expected, cells expressing PKCl RNAi or PKCל RNAi formed significantly fewer colonies in soft agar than cells expressing NT RNAi (Figure 1B). Similarly, cells expressing $\mathrm{PKC}$ r RAi or PKC $\zeta$ RNAi exhibited significantly reduced invasion through matrigel in a transwell invasion assay, compared to Panc1 cells expressing NT RNAi (Figure 1C). Interestingly, expression of exogenous $\mathrm{PKCl}$, but not $\mathrm{PKC}_{\zeta}$, was able to significantly reconstitute the effects of $\mathrm{PKCl}$ RNAi on pancreatic cancer cell growth in soft agar and invasion (Figure 1B and 1C). Conversely, expression of exogenous $\mathrm{PKC} \zeta$, but not $\mathrm{PKCl}$, was able to significantly reconstitute the effects of $\mathrm{PKC} \zeta \mathrm{RNAi}$ on pancreatic cancer cell growth in soft agar and invasion (Figure 1B and $1 \mathrm{C}$ ). These data indicate that $\mathrm{PKCl}$ and $\mathrm{PKC} \zeta$ play non-redundant roles in the maintenance of the transformed phenotype of pancreatic cancer cells. Consistent with this observation, we have shown that $\mathrm{PKCl}$ and $\mathrm{PKC} \zeta$ are preferentially coupled to distinct cancer-promoting signaling pathways in pancreatic cancer cells $[2,3]$. Thus we hypothesized that inhibition of expression of both $\mathrm{PKC} \imath$ and $\mathrm{PKC} \zeta$ would reduce the transformed phenotype significantly more than inhibition of either aPKC alone. To test this hypothesis, Panc-1 cells were co-transfected with NT and $\mathrm{PKC}$ RNAi, NT and PKC $\zeta$ RNAi, or PKCi RNAi and PKC $\zeta$ RNAi (Figure 1D), and assessed for anchorage independent growth and cellular invasion. As predicted, cells expressing both $\mathrm{PKCl}$ and $\mathrm{PKC} \zeta \mathrm{RNAi}$ produced significantly fewer colonies in soft agar (Figure $1 \mathrm{E}$ ) and were significantly less invasive (Figure 1F) than $\mathrm{NT}, \mathrm{PKCl}$ or $\mathrm{PKC} \zeta \mathrm{RNAi}$ cells. Taken together, these data demonstrate that $\mathrm{PKCl}$ and $\mathrm{PKC} \zeta$ function in a nonredundant way to promote the transformed phenotype of pancreatic cancer cells.

\section{Par6 knockdown phenocopies aPKC knockdown in pancreatic cancer cells in vitro}

Par6 is a PB1 domain-containing protein that interacts with aPKCs via PB1 domain-mediated interactions [16]. The Par6-aPKC interaction is necessary for the establishment and maintenance of the cell polarity complex in non-transformed cells [17]. Furthermore, the $\mathrm{PB} 1$ domain-mediated interaction of Par6 and $\mathrm{PKCl}$ is required for $\mathrm{PKCl}$ oncogenic signaling in transformed cells $[4,6,18]$. If the functional effects of $\mathrm{PKCl}$ and $\mathrm{PKC} \zeta$ in pancreatic cancer cells require PB1 domainmediated interactions, then Par6 may also be required for the transformed phenotype of pancreatic cancer cells. To assess the role of Par6 in pancreatic cancer cells, Panc-1 and MIA PaCa- 2 cells were stably transfected with control (NT) RNAi or Par6-targeting RNAis (Figure 2A, Panc-1; 2B, MIA PaCa-2). Similar to the effect of knockdown of $\mathrm{PKC} 1$ or $\mathrm{PKC} \zeta$, pancreatic cancer cells expressing Par6 RNAi exhibit significantly reduced anchorage independent growth and cellular invasion, compared to cells expressing NT RNAi (Figure 2C, Panc-1; Figure 2D, MIA PaCa-2). These data indicate that oncogenic signaling of $\mathrm{PKCl}$ and $\mathrm{PKC} \zeta$ requires $\mathrm{PB} 1$ domain-mediated interactions with Par6 and also suggest that a small molecule inhibitor targeting the PB1 domain interactions of aPKCs will significantly reduce pancreatic cancer cell colony formation and invasion.

\section{ATM inhibits aPKC signaling in vitro}

A high throughput screen of FDA-approved drugs identified small molecule inhibitors of PKC1-Par6 PB1-domain interactions [6]. ATM, a gold-containing compound identified with this screen, significantly inhibits PKC1-mediated transformed growth of lung cancer cells, in vitro and in vivo $[4,9,19]$. Gold-containing compounds are known to be thio-reactive, covalently modifying proteins by formation of thio-gold adducts with reactive cysteine residues [20]. ATM selectively modifies the Cys69 residue within the OPCA motif of the $\mathrm{PKC}$ $\mathrm{PB} 1$ domain and disrupts the $\mathrm{PKC}$-Par6 PB1 domain 
A

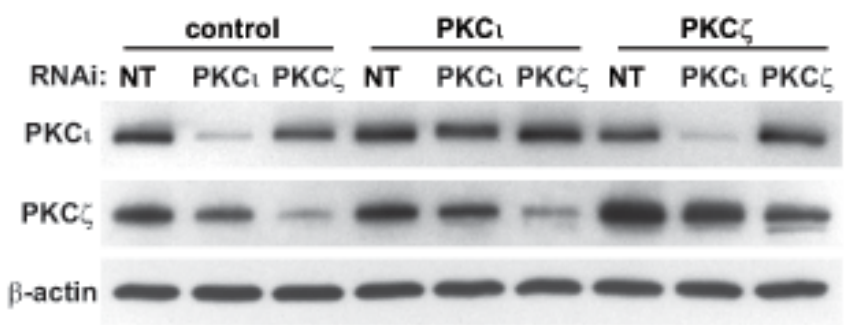

B

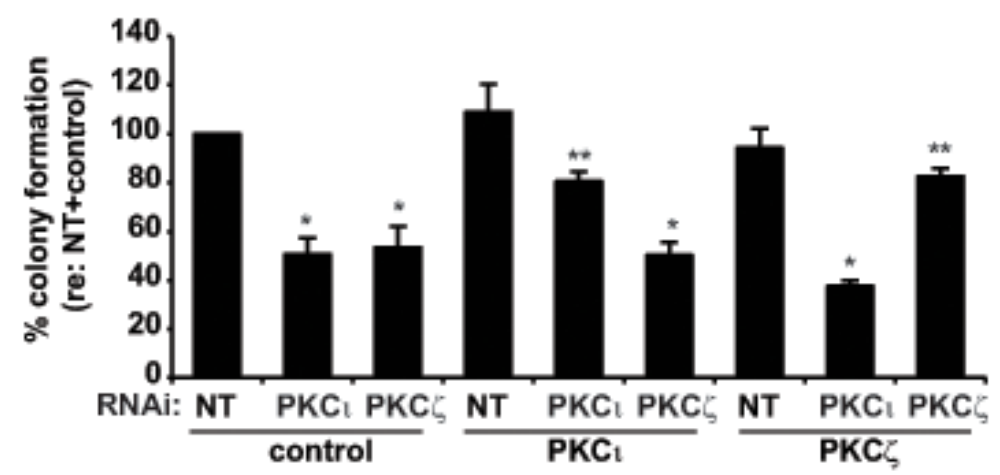

C

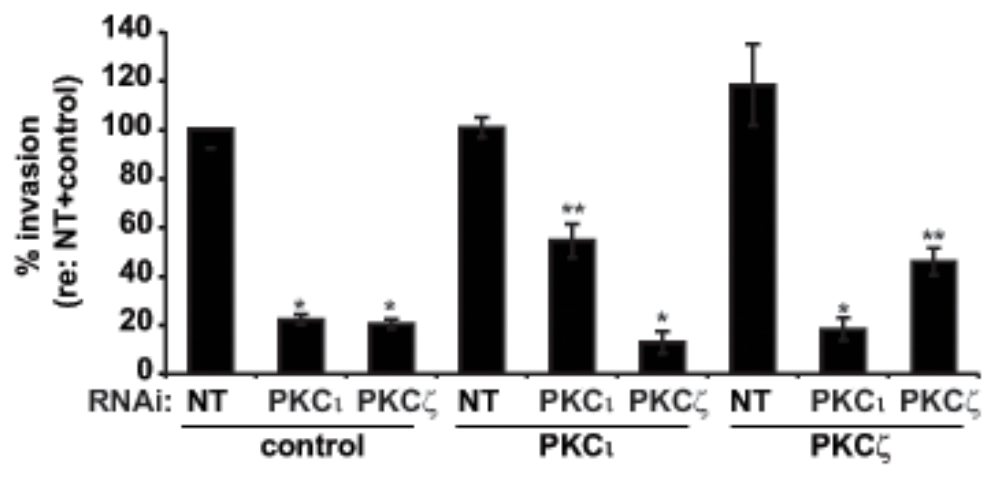

D

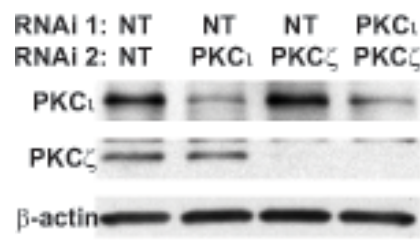

E

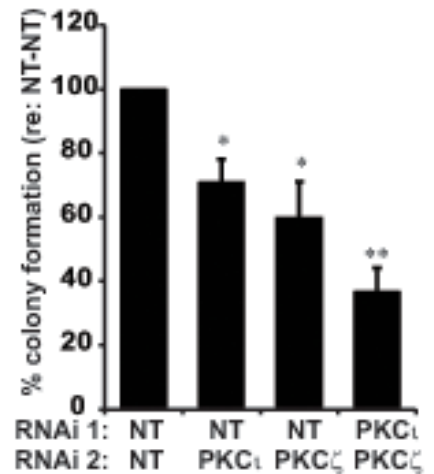

$\mathbf{F}$

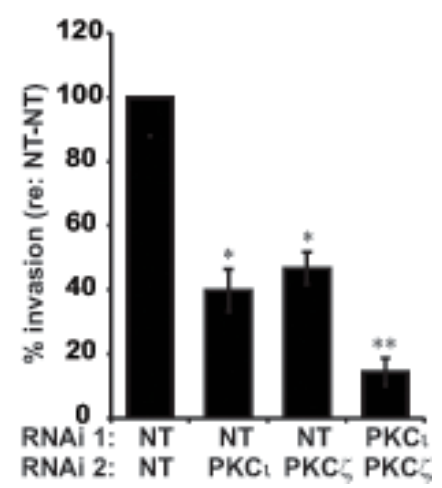

Figure 1: PKCl and PKCל play non-redundant roles in pancreatic cancer cell transformed growth. A-C) Panc-1 cells transfected with lentivirus expressing either control, non-targeting (NT) RNAi, PKC $\zeta$-targeting RNAi, or PKCt-targeting RNAi, and also carrying either control vector ( $\mathrm{pBabe}$ ), or vector expressing $\mathrm{PKCl}$ or $\mathrm{PKC} \zeta$ were assayed for $\mathrm{A}$ ) expression of $\mathrm{PKC}$, $\mathrm{PKC} \zeta$ and actin by immunoblot analyses, B) anchorage-independent growth (colony formation in soft agar), and C) cellular invasion through Matrigel-coated transwell chambers. $\mathbf{B}, \mathbf{C})$ Bars $=$ average of 3 or more replicates $\pm \mathrm{SD}$ and graph is representative of 2 or more independent experiments. ${ }^{*} p$ $<0.05$ vs NT-pBabe; ${ }^{* *} p<0.05$ vs corresponding RNAi + control vector. D-F) Panc-1 cells transfected with two doses of NT RNAi (control), NT and PKCi RNAi, NT and PKC $\zeta$ RNAi, or PKCı and PKC $\zeta$ RNAi were assessed for D) protein expression by immunoblot analysis, E) anchorage-independent growth (colony formation in soft agar), and F) cellular invasion. E, F) Data are expressed relative to $\mathrm{NT}+$ control $\pm \mathrm{SD}, \mathrm{n}=3$ or more, and graph is representative of 2 or more independent experiments. $* p<0.05 v s \mathrm{NT}-\mathrm{NT}$; $* * p<0.05$ vs corresponding $\mathrm{NT}+\mathrm{PKCl}$ or $\mathrm{NT}+\mathrm{PKC} \zeta$ RNAi cell lines. 
interaction in a dose-dependent manner [6]. Interestingly, a similar cysteine residue is found in the PB1 domain of PKCל (Cys68), but not in any other known PB1 domains [15]. The PB1 domain of PKC $\zeta$ binds Par6 in a specific manner (Figure 3A). As predicted by the presence of the ATM-modifiable cysteine within the PKC $\zeta$ PB1 domain, ATM also inhibits the binding of the PB1 domain of PKC $\zeta$ to Par6 in a dose-dependent manner (Figure 3B), with an IC50 value $(3.0 \mu \mathrm{M})$ similar to the IC50 value calculated for disruption of PKC1-Par6 PB1 domain interaction [6].

$\mathrm{PKCl}, \mathrm{PKC} \zeta$ and Par6 are required for pancreatic cancer cell transformed growth; thus, we hypothesized that molecular inhibition of aPKC PB1-domain-mediated interactions will inhibit the transformed signaling of both $\mathrm{PKCl}$ and $\mathrm{PKC}$. Panc-1 and $\mathrm{MIA} \mathrm{PaCa}-2$ pancreatic cancer cell lines were treated with ATM for 48hrs and then assayed for Rac1 activity. ATM treatment inhibits Rac1 activity in pancreatic cancer cells (Figure 4A) consistent with inhibition of $\mathrm{PKCl}$ signaling [2]. Additionally, ATM inhibits STAT3 activation, as detected by a significant decrease in phosphorylation of Y705 (Figure 4B), consistent with inhibition of $\mathrm{PKC} \zeta$ signaling [3] . These data indicate that ATM inhibits pancreatic cancer oncogenic signaling pathways regulated by both $\mathrm{PKCl}$ and $\mathrm{PKC}$ in vitro.

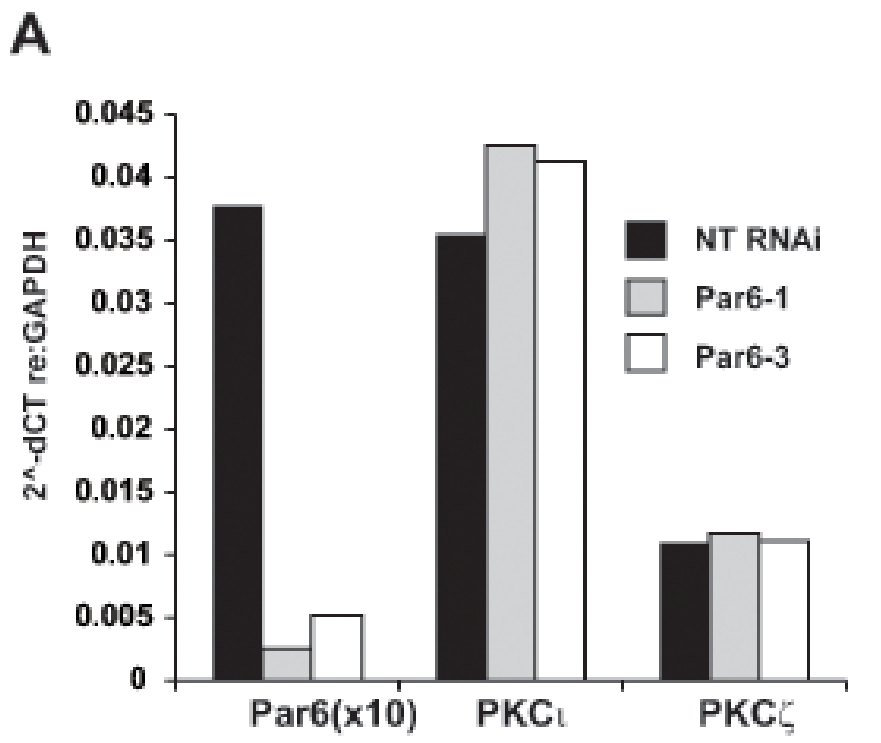

B

C
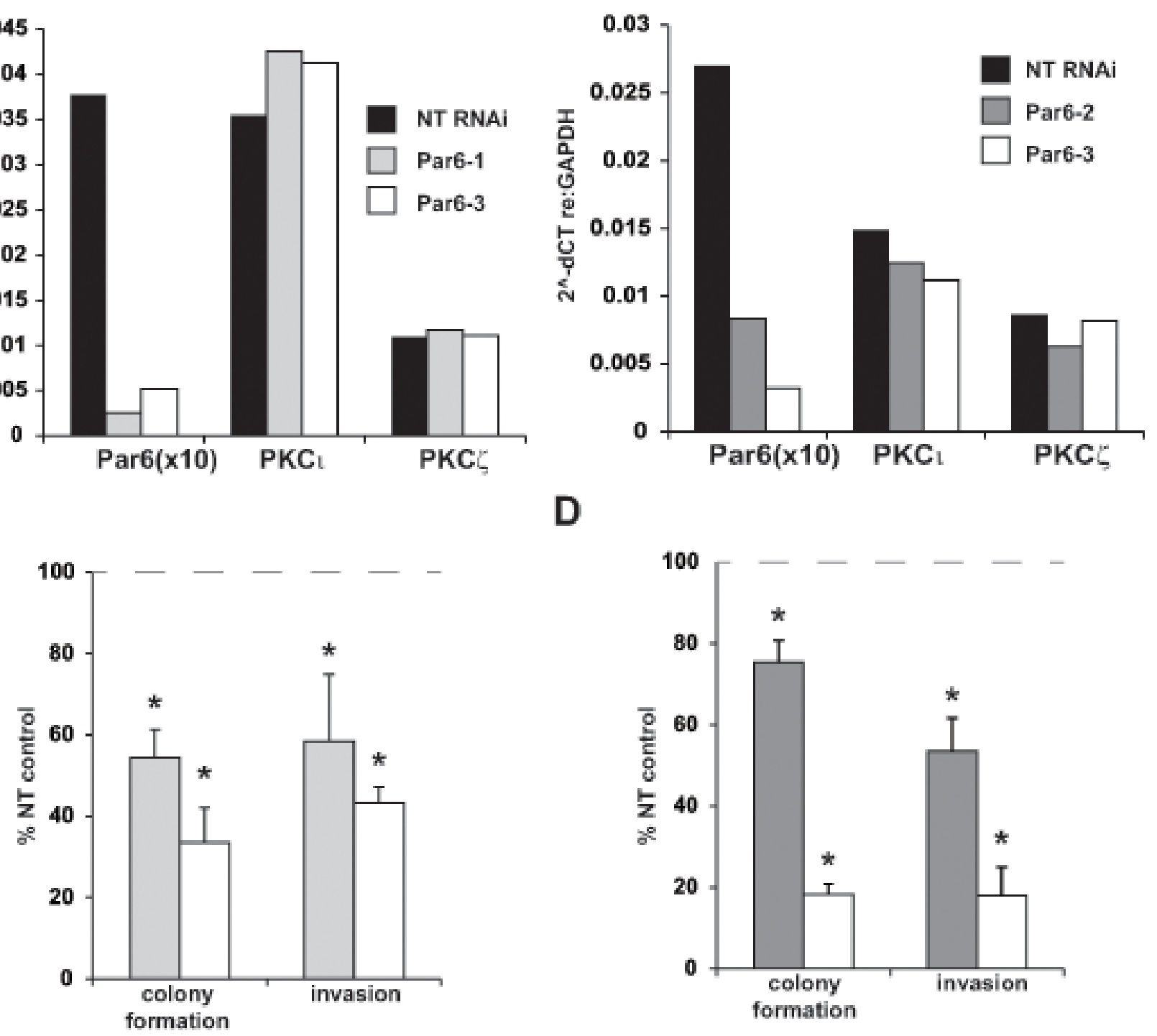

D

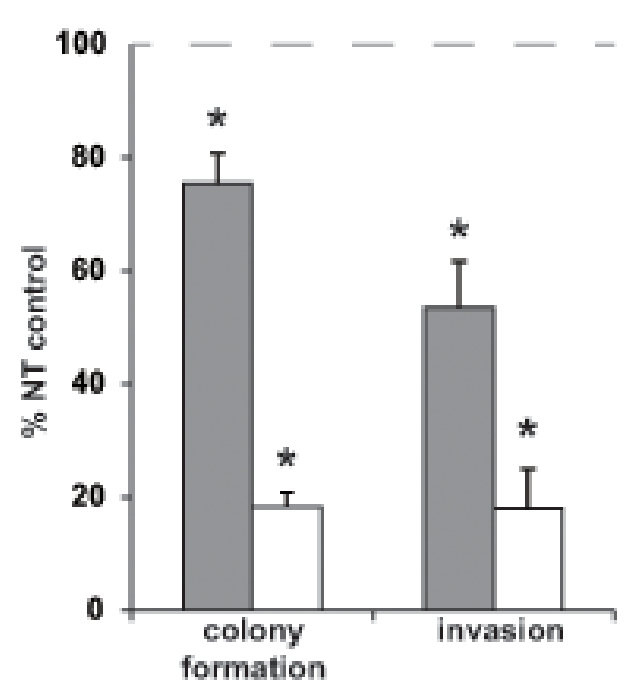

Figure 2: Par6 knockdown phenocopies the effect of aPKC knockdown on pancreatic cancer cell transformed growth and invasion. Panc-1 (A) and MIA PaCa-2 (B) cells stably expressing either NT or Par6-targeted RNAi constructs were evaluated for Par6, PKC1, and PKC $\zeta$ mRNA expression (PKC expression presented as $2^{\wedge}-\mathrm{dCT}$, Par6 expression $=2^{\wedge}$-dCT $\mathrm{x} 10$ for presentation on same scale). C, D) The effect of Par6 knockdown on anchorage-independent growth (soft agar colony formation) and cellular invasion was assessed in (C) Panc-1 and (D) MIA PaCa-2 cells. Bar color indicates Par6-targeting RNAi construct utilized, corresponding to the labels in $\mathrm{A}, \mathrm{B})$. Results are expressed as percent $\mathrm{NT}$ control $\pm \mathrm{SD} \mathrm{n}=3$, and graph is representative of 2 or more independent experiments. $* p<0.05$ vs NT. 


\section{Pharmacological inhibition of aPKCs inhibits the transformed phenotype of pancreatic cancer cells in vitro.}

We have previously shown that knockdown of the aPKCs inhibits the transformed phenotype of pancreatic cancer $[2,3]$; therefore we predicted that a pharmacological inhibitor of aPKC signaling would mimic genetic inhibition. $\mathrm{PKC} 1$ and $\mathrm{PKC} \zeta$ are both expressed in multiple human pancreatic cancer cell lines (Figure 4C), corroborating our published data demonstrating high expression of aPKCs in pancreatic cancer patient tumors $[2,3]$. Panc-1, MIA PaCa-2, and Capan-1 pancreatic cancer cell lines were grown in soft agar in the presence of increasing concentrations of ATM (Figure 4D). ATM inhibited anchorage-independent growth of pancreatic cancer cell lines in a dose-dependent manner. Additionally, ATM inhibited pancreatic cancer cell invasion in a dosedependent manner (Figure 4E). Recently, another thiogold-containing compound, ANF, has been shown to also inhibit $\mathrm{PKC}$ signaling by a mechanism similar to ATM [7]. As predicted, ANF also inhibited anchorageindependent growth (Figure S1A) and invasion (Figure

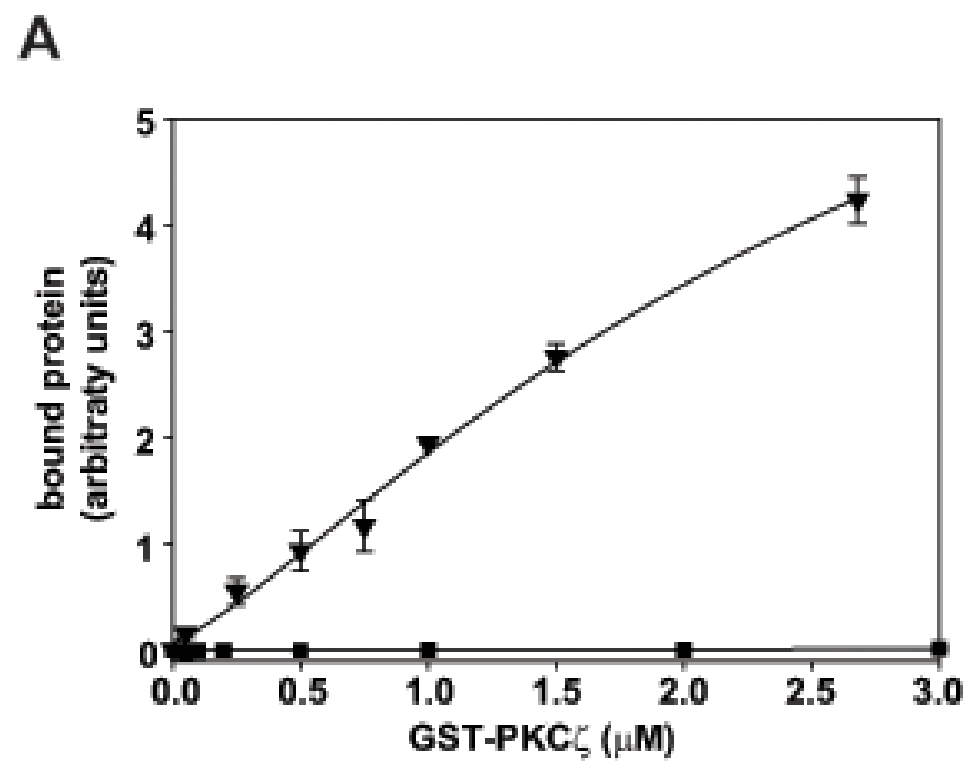

B

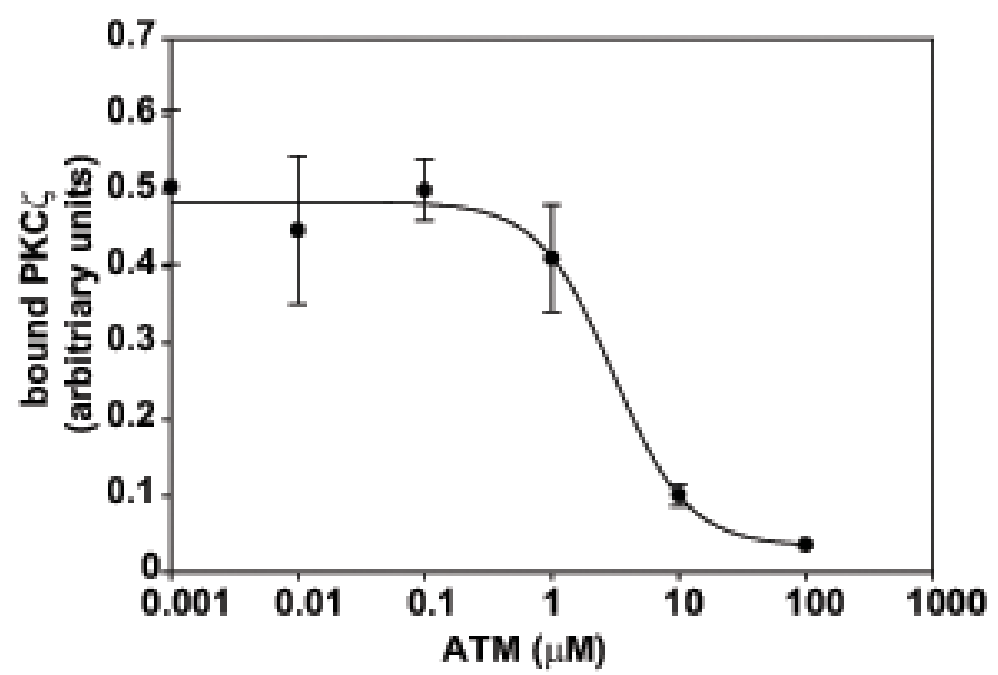

Figure 3: ATM inhibits PB1 domain-mediated interactions. (A) Binding of GST-PKC $(1-113, \boldsymbol{\nabla})$ or GST (-) to Par6. Biotinylated Par6 was bound to streptavidin-coated plates and incubated with increasing concentrations of purified protein. Bound peptide was detected using the MesoScale Discovery detection system. (B) ATM inhibits binding of GST-PKC $\zeta$ to Par6. Biotinylated Par6 bound to streptavidin-coated plates was incubated with $0.4 \mu \mathrm{M}$ of GST-PKC $\zeta$ in the presence of increasing concentrations of ATM. The average of 3 replicates $\pm \mathrm{SD}$ is plotted and graph is representative of 2 or more independent experiments. 
A

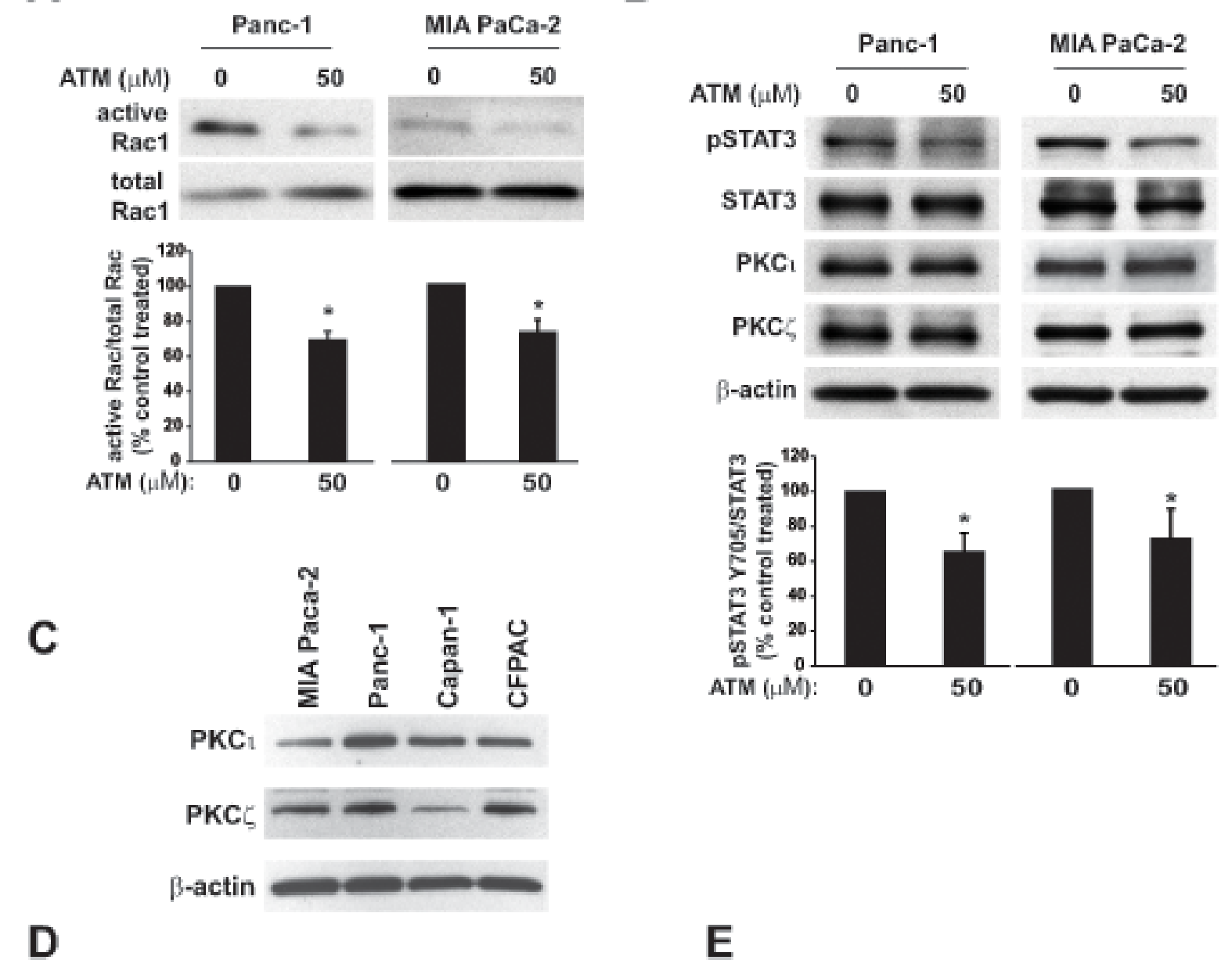

B
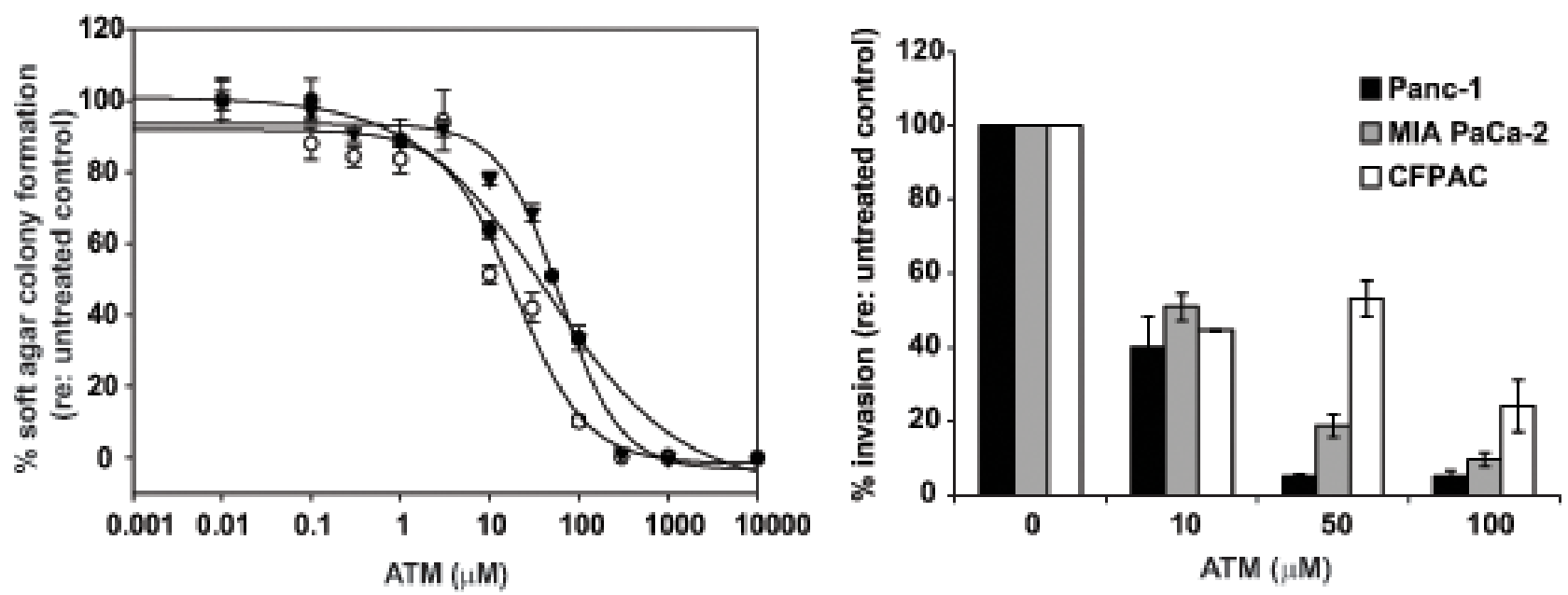

Figure 4: ATM inhibits aPKC downstream signaling and transformed growth in pancreatic cancer cell lines. A) Panc1 and MIA PaCa-2 cells were pre-treated with ATM $(50 \mu \mathrm{M})$ for 48 hrs then assayed for Rac1 activity. Immunoblot analysis of Rac1 in precipitates (active Rac1) and total cellular extracts (total Rac1) is shown. The ratio of active Rac1/total Rac1 is plotted relative to controltreated cells $\left( \pm \mathrm{SD}, \mathrm{n}=4\right.$ independent experiments $\left.{ }^{*} p<0.05\right)$. B) Panc-1 (left) and MIA PaCa-2 (right) cells were treated with ATM $(50$ $\mu \mathrm{M})$ for $48 \mathrm{hrs}$ then subject to immunoblot analysis for expression of p-STAT3, total STAT3, PKCl, PKC $\zeta$, and $\beta$-actin as a loading control. The ratio of active $\mathrm{p}$-STAT3/total STAT3 is plotted relative to control-treated cells $\left( \pm \mathrm{SD}, \mathrm{n}=4\right.$ independent experiments $\left.{ }^{*} p<0.05\right)$. C) Immunoblot analysis of $\mathrm{PKCl}, \mathrm{PKC} \zeta$ and $\beta$-actin expression in MIA PaCa-2, Panc-1, Capan-1 and CFPAC pancreatic cancer cell lines. D) Anchorage-independent growth was assessed in Panc-1 (open circles), MIA PaCa-2 (triangle), and Capan-1 cells (filled circle) in the presence of increasing concentrations of ATM $(\mu \mathrm{M})$. Bar=average of at least 4 replicates \pm SD. E) Cellular invasion was assessed in Panc-1, MIA PaCa-2, and CFPAC cells pre-treated for 48hrs with ATM and in the presence of increasing concentrations of ATM $(\mu \mathrm{M})$. Bar=average of 3 replicates \pm SD. Each panel is representative of 2 or more independent experiments. 
S1B) of pancreatic cancer cell lines in a dose-dependent manner. These data indicate that clinically relevant therapies targeting aPKCs can inhibit the transformed phenotype of pancreatic cancer cells,

We hypothesized that the effect of ATM on the transformed phenotype of pancreatic cancer cells is due to inhibition of both $\mathrm{PKCl}$ and $\mathrm{PKC} \zeta$. We therefore predicted that pancreatic cancer cells expressing either

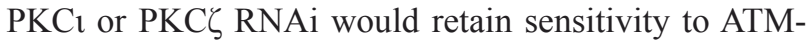
mediated inhibition of anchorage-independent growth and invasion. As predicted, ATM treatment of pancreatic cancer cells expressing $\mathrm{PKCl}$ RNAi further reduced anchorage-independent growth (Figure 5A) and cellular invasion (Figure 5B). Likewise, ATM further reduced anchorage-independent growth (Figure 5A) and invasion of pancreatic cancer cells expressing PKC $\zeta$ RNAi (Figure $5 \mathrm{~B})$. Furthermore, combined knockdown of $\mathrm{PKCl}$ and PKC $\zeta$ by RNAi inhibited invasion to a level similar to the effect of ATM treatment (Figure 5C); these cells were insensitive to additional inhibition by ATM treatment, indicating that $\mathrm{PKCl}$ and $\mathrm{PKC} \zeta$ are the primary cellular targets for ATM-mediated inhibition (Figure 5C).

\section{ATM inhibits pancreatic orthotopic tumor growth, aPKC regulated signaling and metastasis in vivo}

We next investigated the effect of ATM treatment on pancreatic tumor growth using a previously described Panc-1 orthotopic tumor model [2]. Panc-1 cells expressing the firefly luciferase gene (pSIN-Fluc) were injected into the pancreas of nude mice and tumor growth was monitored by bioluminescence (Figure 6A). Mice were treated with either $60 \mathrm{mg} / \mathrm{kg}$ ATM or saline by daily IP injections for 5 weeks. Tumor formation was observed in all mice; however, mice treated with ATM had significantly lower final tumor weight compared to those treated with saline (Figure 6A and 6B). Tumor cell proliferation was also significantly reduced in tumors from ATM-treated mice (Figure 6C). Additionally, tumors from ATM-treated mice had a significant increase in apoptosis (Figure 6D), and tumor necrosis (Figure 6E). These data indicate that the reduced tumor size in ATMtreated mice is due to a combination of decreased tumor cell proliferation and increased cell death. p-ERK1/2 and p-STAT3 levels were reduced in tumors from ATM-treated mice (Figure 6F), consistent with the effects of inhibition of $\mathrm{PKCl}$ [2], and $\mathrm{PKC} \zeta$ [3], respectively, demonstrating target engagement in vivo. Furthermore, significantly fewer metastases to distal organs were detected in mice treated with ATM compared to saline-treated mice (Figure $6 \mathrm{G})$. These data demonstrate that pharmacological inhibition of aPKCs is a promising therapeutic approach for the treatment of pancreatic cancer.

\section{DISCUSSION}

We have previously shown that both $\mathrm{PKCl}$ and $\mathrm{PKC} \zeta$ play promotive roles in the transformed growth of pancreatic cancer cells in vitro and in vivo $[2,3]$. In the present study, we first demonstrate that $\mathrm{PKCl}$ and $\mathrm{PKC} \zeta$ play non-redundant roles in pancreatic cancer, and that knockdown of both $\mathrm{PKCl}$ and $\mathrm{PKC} \zeta$ results in significant, additive inhibition of the transformed phenotype compared to inhibition of either aPKC alone. These data extend our previous observations that $\mathrm{PKCl}$ and $\mathrm{PKC} \zeta$ are preferentially coupled to distinct procancer signaling pathways in pancreatic cancer $[2,3]$. $\mathrm{PKC} \mathrm{l}$ promotes the transformed phenotype of pancreatic cancer, at least in part, via activation of Rac1-MEK/ERK signaling [2], whereas a major mechanism by which PKCל regulates pancreatic cancer cell transformed growth is via promotion of STAT3 activation [3]. Therefore, an inhibitor targeting both aPKCs would simultaneously inhibit two critical oncogenic signaling pathways in pancreatic cancer.

In addition to pancreatic cancer, $\mathrm{PKCl}$ has been shown to play an oncogenic/cancer-promoting role in lung, ovarian, and colon cancers (reviewed in Murray et al. [21]). The oncogenic role of $\mathrm{PKCl}$ has been particularly well-described in lung cancer, where $\mathrm{PKC}$ is required for oncogenic K-ras-induced tumor initiation and progression in a mouse model of lung cancer, as well as for maintenance of the transformed phenotype of human lung cancer cells [5, 18, 22]. Further investigation of the mechanism of $\mathrm{PKCl}$ oncogenic signaling in lung cancer revealed that $\mathrm{PKCl}$ mediates its effects by forming an oncogenic signaling complex through interactions involving its PB1-domain [4, 9, 19].

A high throughput screen of a library of FDAapproved drugs for compounds that disrupted the PB1 domain-mediated interaction between $\mathrm{PKCl}$ and Par6 identified several gold-containing compounds, used clinically for the treatment of rheumatoid arthritis [6]. One of the gold-containing drugs identified as a $\mathrm{PKC}$ PB1 domain interaction disruptor, ATM, inhibits lung cancer cell transformed growth in vitro and in vivo $[6,19]$. FDA-approved gold-containing small molecules have been used clinically for over 30 years for the treatment of rheumatoid arthritis [23]. While the mechanism of action of gold-containing drugs in the treatment of rheumatoid arthritis has never been fully elucidated, the inhibition of mitochondrial thioredoxin reductase has been suggested as a mediator of therapeutic response [20]. Thioredoxin reductase is considered an important target for immunosuppression in the treatment of rheumatoid arthritis, since thioredoxin reductase is highly expressed in the synovial cells of rheumatoid arthritis patients [24]. Other hypothesized mechanisms of action of goldcontaining compound-induced immunosuppression are inhibition of IL-6/JAK/STAT3 signaling and inhibition of NF-кB [25-27]. Recent evaluation of gold-containing 

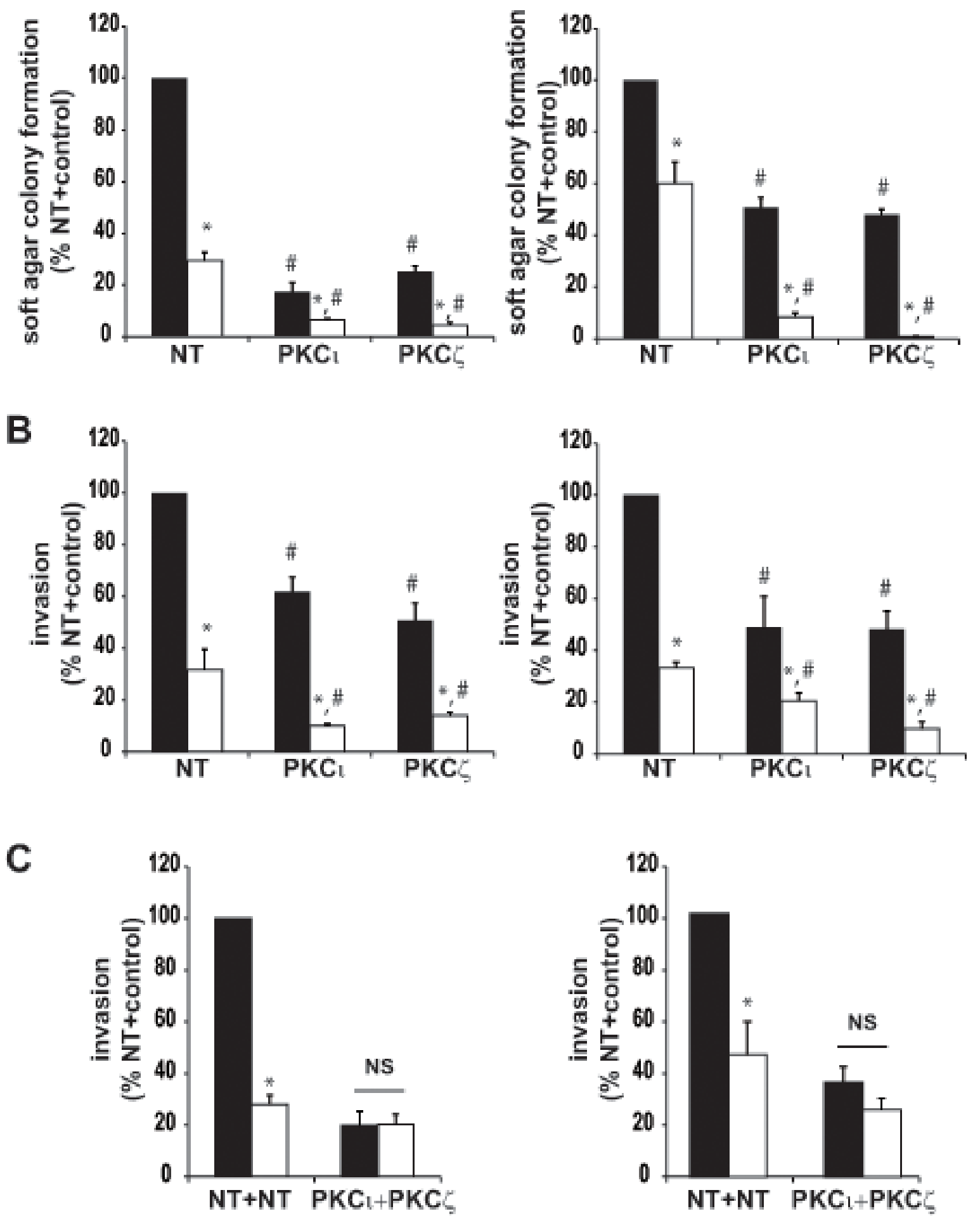

Figure 5: Effect of aPKC knockdown on pancreatic cancer cell sensitivity to ATM inhibition of the transformed phenotype. A) Anchorage-independent growth was assessed in Panc-1 (left), and MIA PaCa-2 (right), cells stably expressing NT, PKC1, or PKC $\zeta$ RNAi in the presence of control diluent (filled bars) or $50 \mu \mathrm{M}$ of ATM (open bars). Bar=average of at least 4 replicates \pm SD. B) Cellular invasion was assessed in Panc-1 (left), and MIA PaCa-2 (right), cells stably expressing NT, PKCl, or PKC $\zeta$ RNAi in the presence of control diluent (filled bars) or $50 \mu \mathrm{M}$ of ATM (open bars). Bar=average of 3 replicates $\pm \mathrm{SD} .{ }^{*} p<0.05$ versus the same RNAi construct with control treatment, $\# p<0.05$ versus NT RNAi with same treatment. C) Cellular invasion was assessed in Panc-1 (left), and MIA PaCa-2 (right), transfected with two doses of NT RNAi (control), or PKC 1 and PKC $\zeta$ RNAi, in the presence of control diluent (filled bars) or $50 \mu \mathrm{M}$ of ATM (open bars). Bar=average of 3 replicates $\pm \mathrm{SD},{ }^{*} p<0.05$ versus the same RNAi construct with control treatment, NS=difference is not statistically significant. Each panel is representative of 2 or more independent experiments. 
A
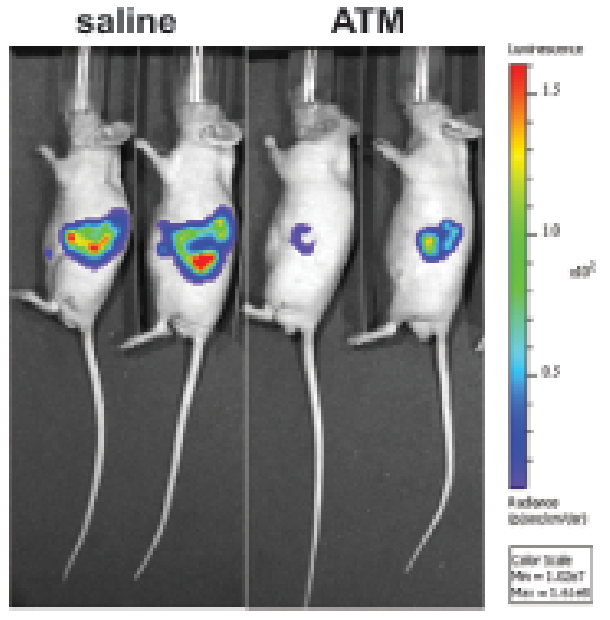

B

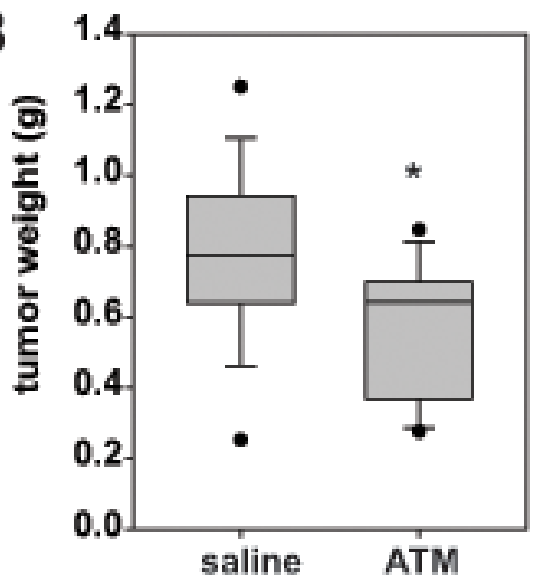

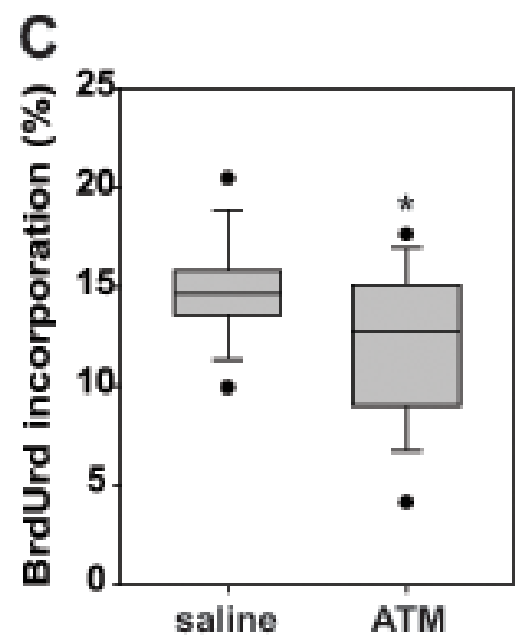

F

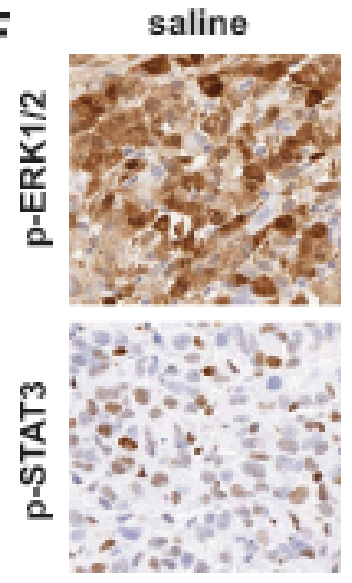

D

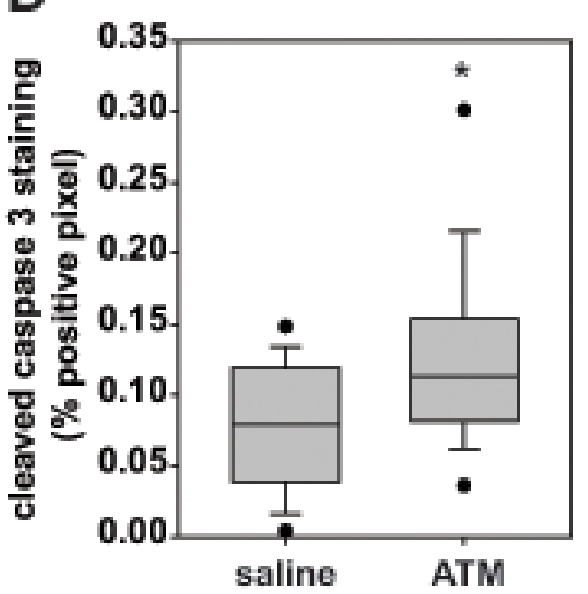

E

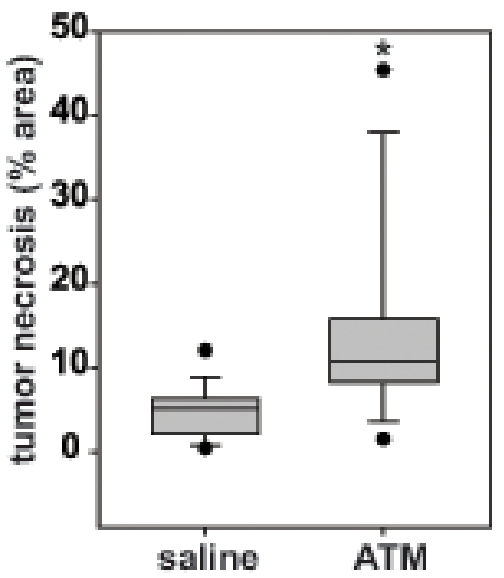

ATM

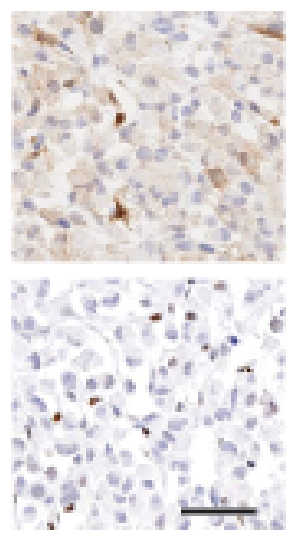

G

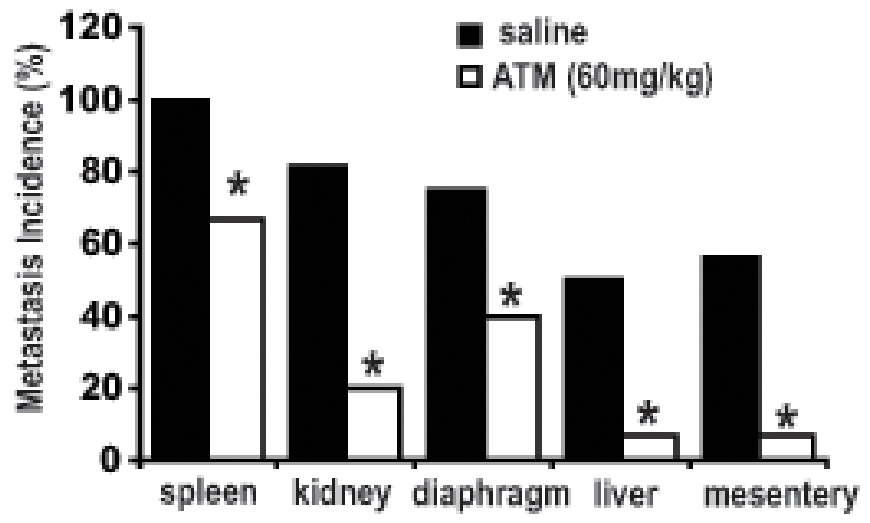

Figure 6: ATM inhibits Panc-1 tumor size and aPKC signaling pathways in vivo. A) Tumor growth was monitored by bioluminescence (total flux, photons per second) detected by IVIS imaging of orthotopic Panc-1 pancreatic tumors in live, anesthetized mice. Representative bioluminescent imaging of mice with orthotopic Panc-1 pancreatic tumors treated daily with either saline or 60mg/ $\mathrm{kg}$ ATM is shown. Mice were sacrificed after 4 weeks of treatment and tumors were analyzed for the following characteristics: B) final tumor weight, C) tumor BrdUrd incorporation, D) cleaved caspase-3, and E) tumor necrosis as described in Materials and Methods. F) Mice carrying orthotopic Panc-1 tumors were treated with daily injections of saline or $60 \mathrm{mg} / \mathrm{kg}$ ATM for 7 days and harvested 24 hours after the final injection. Tumors were assessed for p-ERK1/2 (top panels), and p-STAT3 (bottom panels) by IHC staining. Bar=100 $\mu$ m. G) Mice treated for 4 weeks with ATM or saline were evaluated for metastases to distal organs as described in Materials and Methods. The percentage of mice in each treatment group with confirmed metastasis to each distal organ is plotted, $\mathrm{n}=16$ per group, Difference in metastasis to each organ was analyzed by Fisher Exact test and $* p<0.05$ was considered significant. 
rheumatoid arthritis drugs for anti-cancer properties has revealed both apoptotic and cytotoxic effects in various cancer cell types, including breast, ovarian and leukemia [28-31]. These studies investigated multiple potential targets for the anti-cancer effects of these drugs, including inhibition of thioredoxin reductase, STAT3 signaling, proteasome-associated deubiquitinases and telomerase [28-31]. While the mechanism of action of gold-containing drugs in most cancer types remains poorly understood, mechanistic studies in lung cancer cells demonstrate that ATM-mediated inhibition of transformed growth requires the gold-modifiable Cys69 residue in the $\mathrm{PKCl} \mathrm{PB} 1$ domain, demonstrating the necessity for $\mathrm{PKC}$ inhibition for the anti-tumor effects of ATM in lung cancer cells [4, $6,19]$. Furthermore, sensitivity to ATM-mediated growth inhibition in lung cancer cells correlates directly with expression of $\mathrm{PKCl}$ and Par6 but not to other proposed ATM targets such as thioredoxin reductases [19].

Similar to $\mathrm{PKCl}$, the $\mathrm{PB} 1$ domain of $\mathrm{PKC} \zeta$ contains a cysteine residue at position 68; thus we hypothesized that ATM would disrupt PKC $\zeta$ PB1 domain-mediated interactions in a similar manner to its effect on PKC1-Par6 interactions $[4,16]$. Indeed, in vitro analysis demonstrated that ATM disrupts the interaction of the PKC $\zeta$ PB1 domain and Par6 with an IC50 value similar to that characterized for a PKC1-Par6 interaction [4, 6]. We next evaluated the therapeutic potential of pharmacological inhibition of both $\mathrm{PKC}$ and $\mathrm{PKC} \zeta$ in pancreatic cancer cells. ATM inhibited both $\mathrm{PKCl}_{1}$ and $\mathrm{PKC} \zeta$-regulated signaling pathways in pancreatic cancer cells in vitro and in vivo. Likewise, ATM inhibited the transformed growth of pancreatic cancer cells in vitro and in vivo, similar to the effect of genetic inhibition of $\mathrm{PKCl}$ and $\mathrm{PKC} \zeta$. These data demonstrate that ATM is able to simultaneously target both $\mathrm{PKCl}$ and $\mathrm{PKC} \zeta$, thereby inhibiting two critical pathways in pancreatic cancer (Figure 7).

In addition to ATM, ANF, a gold-containing drug used to treat rheumatoid arthritis, has been evaluated for anti-cancer activity. ANF was shown to inhibit $\mathrm{PKCl}$ signaling in ovarian cancer cells, and inhibit $\mathrm{PKCl}$ mediated transformed growth, similar to the effect of ATM in lung cancer cells $[4,7]$. Previously published structureactivity studies of gold (I) complexes demonstrated that derivatives containing a phosphine group (ANF but not ATM) have increased cytotoxic effects against cancer cells [32], and that in vitro cytotoxicity and in vivo antitumor effects are related to the increased lipophilicity provided by the phosphine group [33]. Likewise, we observed that ANF inhibited anchorage-independent growth and invasion in pancreatic cancer cells with a greater potency

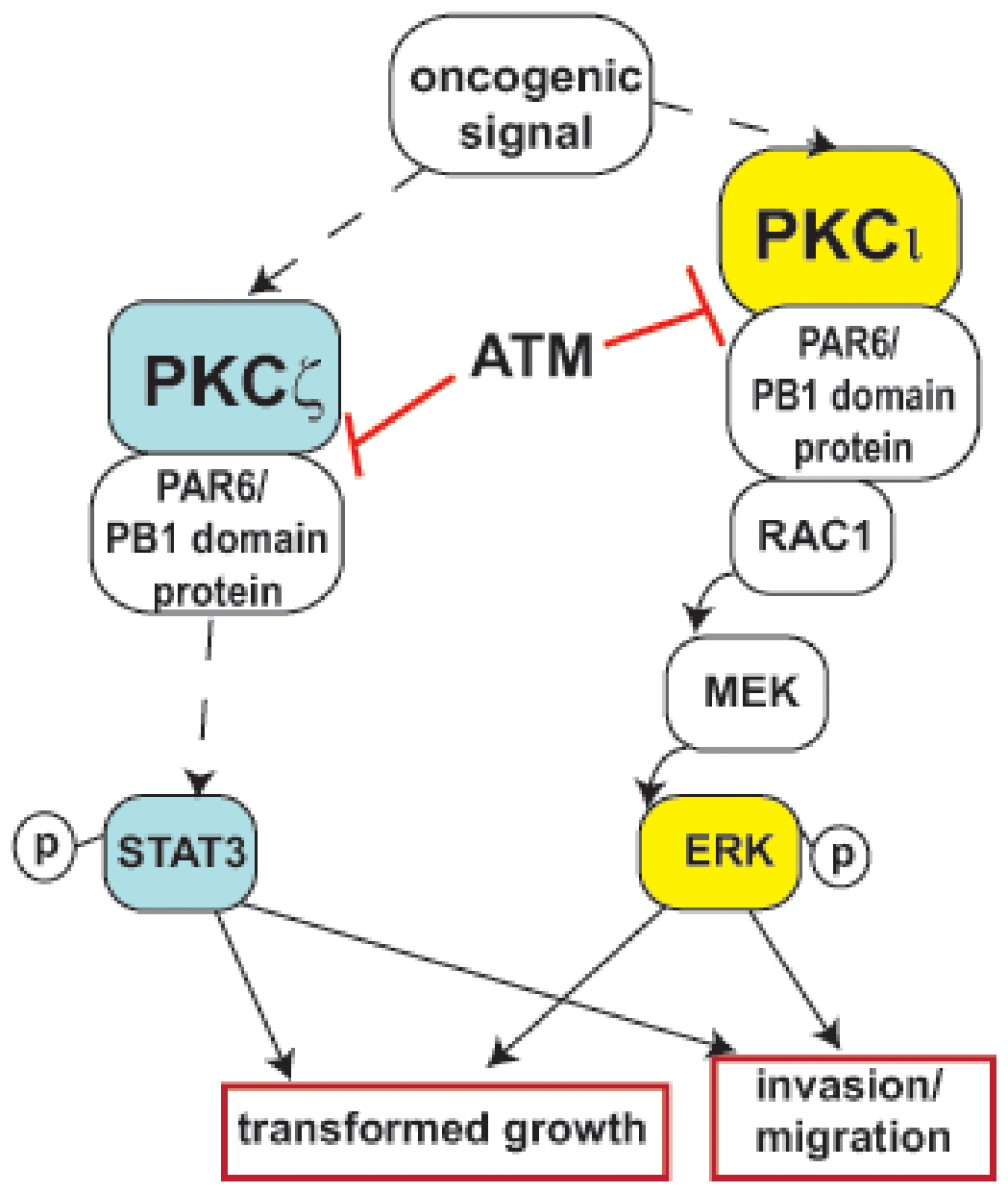

Figure 7: Summary of ATM-mediated inhibition of aPKC signaling. 
than ATM (Figure 4 and Supplemental Figure 1). Taken together, we show that gold-containing compounds inhibit the transformed growth of pancreatic cancer cell lines by inhibiting $\mathrm{PKCl}$ - and $\mathrm{PKC}$-regulated signaling pathways. These data further support the therapeutic potential of targeting the aPKCs in pancreatic cancer.

Unlike several other cancers, the lethality of pancreatic cancer has gradually increased over the past decade [1] and thus the need for more successful treatments has grown more critical. Our previous work identified the aPKCs, $\mathrm{PKCl}$ and $\mathrm{PKC} \zeta$, as potential therapeutic targets for the treatment of pancreatic cancer. ATM and ANF have been shown to specifically inhibit $\mathrm{PKC} r$ signaling and tumor growth in lung [6, 19] and ovarian cancer models [7]. Recently, ATM was evaluated in a phase I clinical trial of patients with advanced nonsmall cell lung cancer, ovarian, and pancreatic cancers [34]. This study identified a maximum tolerated dose (MTD) for ATM corresponding to serum gold levels above the level necessary to successfully inhibit aPKC signaling and reduce tumor growth in preclinical models, indicating the clinical relevance of ATM and gold-containing compounds for the treatment of cancers in which aPKC signaling promotes the cancer phenotype $[19,34]$. In the present study, we provide pre-clinical evidence that ATM disrupts aPKC signaling and inhibits the transformed phenotype in pancreatic cancer, and demonstrate that aPKCs are the major target of ATM-mediated inhibition of the transformed phenotype. Therefore aPKC inhibitors may be a promising new treatment option for pancreatic cancer patients. Future clinical studies are necessary to determine the efficacy of ATM and ANF for the treatment of pancreatic cancer.

\section{MATERIALS AND METHODS}

\section{Reagents and cell culture}

Human pancreatic cancer cell lines were purchased from American Type Culture Collection and all experiments were performed with cells passaged less than 6 months. Human pancreatic cancer cell lines were maintained in a $5 \% \mathrm{CO}_{2}$ humidified tissue culture incubator in DMEM (Panc-1, MIA PaCa-2, and CFPAC) or IMDM (Capan-1) with 10\% (fetal bovine serum) FBS as recommended by American Type Culture Collection. Antibodies were obtained from the following sources: PKC $\zeta$, $\beta$-actin, phospho-STAT3 (Y705), STAT3, phosphoERK1/2, ERK1/2 and cleaved caspase-3 (all from Cell Signaling Technologies), $\mathrm{PKCl}$ (BD Transduction Laboratories), and 5-bromo-2'-deoxyuridine (BrdUrd) (DakoCytomation). GST-PAK1-p21 binding domain (PBD) beads were generated as previously described [8]. Briefly, BL21 bacteria harboring the fusion protein GST- human PAK1 PBD in the pGEX2TK vector (received from Howard Crawford) were induced with IPTG then lysed, purified, and mixed with Glutathione Sepharose 4B beads. ATM was purchased from Akon Pharmaceuticals and Sigma, ANF was purchased from Santa Cruz Biotechnology, Inc.

\section{RNA isolation, quantitative real-time PCR (qPCR) and analysis}

Total RNA was isolated using RNAqueous Isolation Kit (Ambion) according to the manufacturer's protocols. TaqMan ${ }^{\circledR}$ Gene Expression Assay primer and probe sets (Applied Biosystems) were used for real-time, quantitative PCR (qPCR) analysis of GAPDH (Hs99999905 m1),

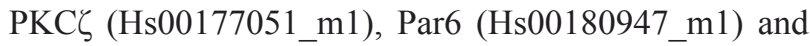
18S (Hs99999901_s1). Forward and reverse primer and probe sequences for $\mathrm{PKCl}$ were previously described [2]. qPCR analysis was carried out using $10 \mathrm{ng}$ of cDNA or 2 ng cDNA (18S) on an Applied Biosystems 7900 thermal cycler. Data was evaluated using the SDS 2.3 software package. Gene expression was normalized to GAPDH. All data is expressed as $2^{-(C T \text { (target)- } C T \text { (endogenous reference)) }}$.

\section{Knockdown and re-expression of human $\mathrm{PKCl}$ and $\mathrm{PKC} \zeta$ genes}

Lentiviral vectors carrying short hairpin RNA interference (RNAi) constructs targeting $\mathrm{PKCl}, \mathrm{PKC} \zeta$, or Par6 were generated and used to obtain stable transfectants as described previously $[3,9]$. PKCı RNAi construct - GCCTGGATACAATTAACCATT; PKC $\zeta$ RNAi construct - GTTGTTCCTGGTCATTGAGTA; Par6 RNAi constructs - TCAGTCATAGACGTGGACCTA (Par6-1), TGGACGTGCTACTTGGCTATA (Par6-2) and GCTGAGCCTGATAGTGACGAT (Par6-3). The cDNA for human PRKCZ (NM_002744.4) was cloned into the pBABE retroviral expression vector. Cells were stably transfected with pBabe, pBabe- $\mathrm{PKCl}$ [10] or pBabe$\mathrm{PKC}$. Cell populations carrying the viral constructs were selected and maintained by inclusion of puromycin in the culture media.

\section{Anchorage-independent growth assays}

Pancreatic cancer cells $\left(7 \times 10^{3}\right)$ were plated in soft agar and assessed for anchorage-independent growth as described previously $[2,3]$.

\section{Cellular invasion assay}

Cellular invasion was assayed using Matrigel-coated invasion chambers (BD Biosciences) according to the manufacturer's protocol. Briefly, $5 \times 10^{4}$ human pancreatic 
cancer cells were plated in serum-free media in the top chamber, and media containing $2.5 \%$ FBS was used as the chemo-attractant in the bottom chamber. Cells were allowed to invade for $18 \mathrm{hrs}$ at $37^{\circ} \mathrm{C}$ and then cells were fixed, stained and quantitated as previously described [11].

\section{PKC $\zeta$ and Par6 binding assay}

Streptavidin-coated 96-well singleplex plates (MesoScale Discovery, Rockville, MD) were blocked with $5 \%$ non-fat dry milk for $1 \mathrm{hr}$ at room temperature. Wells were washed twice with PBS/Tween. After washing, recombinant biotinylated human Par6 protein was bound by incubation at room temperature for $1 \mathrm{hr}$. GST-tagged PKC $\zeta$ N-terminal fragment (amino acids 1-113) or GST control were purified via B-PER GST Protein Purification Kit (Pierce, Rockford, IL) and labeled with Sulfo-Tag NHS-Ester for $2 \mathrm{hrs}$. After removing the free SulfoTag NHS-Ester reagents, increasing concentrations of purified protein was added to Par6 coated plates. After overnight incubation at $4^{\circ} \mathrm{C}$, the wells were washed once with PBS/Tween and $150 \mu 1$ of $1 x$ Reading Buffer was added. Binding to Par6 was determined by detection of electrochemiluminescent signal emitted from the SulfoTag $(620 \mathrm{~nm})$, using a SECTOR Imager 2400 (Meso Scale Discovery). To determine the effect of ATM on PKC $\zeta$ binding to Par6, $0.4 \mu \mathrm{M}$ of GST-tagged PKC $\zeta$ (1-113) was added to each well along with ATM to achieve the desired final concentration as indicated in the figure, and binding detected as described.

\section{Rac1 activity assay}

Pancreatic cancer cells treated with saline or ATM were harvested and assayed for the presence of active, GTP-bound Rac1. Rac1-GTP was precipitated from cell extracts with PAK-1 PBD agarose beads as described previously [10].

\section{Orthotopic tumor model}

Panc-1 human pancreatic cancer cells $\left(\begin{array}{lll}1 & \mathrm{x} & 10^{6}\end{array}\right)$ carrying the pSIN-Fluc luciferase expression vector were mixed with growth factor-reduced Matrigel (Becton Dickinson) and injected into the proximal pancreas $(n=15$ mice/group respectively) of 4-6 week old athymic nude mice. All surgeries were performed under isoflurane anesthesia, and mice were administered buprenorphine as an analgesic immediately before and $\sim 18$ hours after the surgery to minimize animal discomfort. Tumor-bearing mice were monitored daily for signs of distress and twice weekly for weight loss. Mice received IP injections of saline or $60 \mathrm{mg} / \mathrm{kg}$ ATM 5 days/week, beginning on day 8 and continuing until one day prior to sacrifice. For weekly imaging, mice were injected intraperitoneally (IP) with $150 \mathrm{mg} / \mathrm{kg}$ body weight D-Luciferin solution (Xenogen), anesthetized with isoflurane and imaged using a bioluminescence imaging system (IVIS Imaging Spectrum System). Bioluminescence was calculated using IVIS Imaging Spectrum software. Week 1 bioluminescence was used to distribute mice evenly between saline and ATM treatment groups. One hour prior to sacrifice, mice were injected IP with $100 \mu \mathrm{g} / \mathrm{g}$ BrdUrd. All of the animal experiments were approved by the Mayo Clinic Institutional Animal Care and Use Committee.

\section{Orthotopic tumor analysis}

Formalin-fixed pancreatic tumors were analyzed by immunohistochemical (IHC) detection of BrdUrd incorporation, as described previously [12-14]. Orthotopic pancreatic tumors were evaluated for apoptosis by IHC detection of cleaved caspase- 3 as previously described [14]. Tumor necrosis was identified in H\&E stained tissue as described previously [3]. Tumors were fixed by perfusion with $10 \%$ paraformaldehyde at the time of harvest for detection of expression of p-ERK1/2 and p-STAT3 by IHC analysis. p-ERK $1 / 2$ and p-STAT3 staining was visualized using the Envision Plus AntiRabbit Labeled Polymer-HRP (Dako). Images were captured and analyzed using Aperio Spectrum software. Staining with only the secondary antibody served as a negative control. Tumor metastases were identified by detection of bioluminescence by ex vivo IVIS imaging of isolated organs and confirmed by examination of $\mathrm{H} \& \mathrm{E}$ stained tissue sections of the distal organs.

\section{Statistical analysis}

Two-way ANOVA, Student $t$-test or Fisher Exact (tumor metastasis) were used to evaluate the statistical significance of the results. $p<0.05$ was considered statistically significant.

\section{ACKNOWLEDGEMENTS}

We thank Dr. Yasuhiro Ikeda of the Mayo Clinic (Rochester, MN) for providing the pSIN-Fluc vector and the Mayo Clinic RNA Interference Technology Resource for RNAi reagents. We thank Brandy Edenfield of the Mayo Cancer Biology Histology Facility, Lee Jamieson, Verline Justilien and Julie Bray for excellent technical assistance. We thank Dr. Howard Crawford for the GSTPAK1-PBD construct.

\section{FINANCIAL SUPPORT}

NIH/NCI R03 CA143164, NIH/NCI R01CA140290 (NRM), NIH/NCI F31 CA168117 (AMB), NIH/NCI 
R01 CA081436-16 (APF), The Mayo Clinic Foundation (NRM, APF). APF is the Monica Flynn Jacoby Endowed Professor of Cancer Research.

\section{CONFLICT OF INTEREST STATEMENT}

A provisional patent related to this research has been filed (MSB, APF, NRM; Methods and Materials for treating pancreatic cancer, US Patent Application \#20110190390). All other authors have nothing to disclose.

\section{Abbreviations}

Auranofin, ANF; Aurothiomalate, ATM; BrdUrd, 5-bromo-2 '-deoxyuridine; H\&E, hematoxylin and eosin; IHC, immunohistochemical analysis; PKC, protein kinase $\mathrm{C}$; qPCR, quantitative real time polymerase chain reaction; RNAi, RNA interference; Signal transducer and activator of transcription 3, STAT3;

\section{REFERENCES}

1. Siegel R, Naishadham D and Jemal A. Cancer statistics, 2012. CA Cancer J Clin. 2012; 62:10-29.

2. Scotti ML, Bamlet WR, Smyrk TC, Fields AP and Murray NR. Protein kinase $\mathrm{C} t$ is required for pancreatic cancer cell transformed growth and tumorigenesis. Cancer Research. 2010; 70:2064-2074.

3. Butler AM, Scotti Buzhardt ML, Li S, Smith KE, Fields $\mathrm{AP}$ and Murray NR. Protein kinase $\mathrm{C}$ zeta regulates human pancreatic cancer cell transformed growth and invasion through a STAT3-dependent mechanism. PLoS ONE. 2013; 8:e72061.

4. Erdogan E, Lamark T, Stallings-Mann M, Lee J, Pellecchia M, Thompson EA, Johansen T and Fields AP. Aurothiomalate inhibits transformed growth by targeting the PB1 domain of protein kinase Ciota. J Biol Chem. 2006; 281:28450-28459.

5. Regala RP, Davis RK, Kunz A, Khoor A, Leitges M and Fields AP. Atypical protein kinase $\mathrm{Cl}$ is required for bronchioalveolar stem cell expansion and lung tumorigenesis. Cancer Research. 2009; 69:7603-7611.

6. Stallings-Mann M, Jamieson L, Regala RP, Weems C, Murray NR and Fields AP. A novel small-molecule inhibitor of protein kinase Ciota blocks transformed growth of non-small-cell lung cancer cells. Cancer Res. 2006; 66:1767-1774.

7. Wang Y, Hill KS and Fields AP. PKCiota Maintains a Tumor-initiating Cell Phenotype That Is Required for Ovarian Tumorigenesis. Mol Cancer Res. 2013; 11:16241635.

8. Ardito CM, Gruner BM, Takeuchi KK, LubesederMartellato C, Teichmann N, Mazur PK, Delgiorno KE,
Carpenter ES, Halbrook CJ, Hall JC, Pal D, Briel T, Herner A, Trajkovic-Arsic M, Sipos B, Liou GY, et al. EGF receptor is required for KRAS-induced pancreatic tumorigenesis. Cancer Cell. 2012; 22:304-317.

9. Frederick LA, Matthews JA, Jamieson L, Justilien V, Thompson EA, Radisky DC and Fields AP. Matrix metalloproteinase-10 is a critical effector of protein kinase C1-Par6 $\alpha$-mediated lung cancer. Oncogene. 2008; 27:48414853.

10. Murray NR, Jamieson L, Yu W, Zhang J, Gokmen-Polar Y, Sier D, Anastasiadis P, Gatalica Z, Thompson EA and Fields AP. Protein kinase $\mathrm{C}\{$ iota $\}$ is required for Ras transformation and colon carcinogenesis in vivo. J Cell Biol. 2004; 164:797-802.

11. Zhang J, Anastasiadis, P. Z., Liu, Y., Thompson, E.A. and Fields, A.P. Protein Kinase C BII Induces Cell Invasion Through a Ras/MEK-, PKCiota/RAC 1-dependent Signaling Pathway. J Biol Chem. 2004; 279:22118-22123.

12. Calcagno SR, Li S, Colon M, Kreinest PA, Thompson EA, Fields AP and Murray NR. Oncogenic K-ras promotes early carcinogenesis in the mouse proximal colon. Int J Cancer. 2008; 122:2462-2470.

13. Murray NR, Weems C, Chen L, Leon J, Yu W, Davidson LA, Jamieson L, Chapkin RS, Thompson EA and Fields AP. Protein kinase $\mathrm{C}$ betaII and TGFbetaRII in omega-3 fatty acid-mediated inhibition of colon carcinogenesis. $\mathrm{J}$ Cell Biol. 2002; 157:915-920.

14. Fields AP, Calcagno SR, Krishna M, Rak S, Leitges M and Murray NR. Protein kinase Cbeta is an effective target for chemoprevention of colon cancer. Cancer Res. 2009; 69:1643-1650.

15. Selbie LA, Schmitz-Peiffer C, Sheng $Y$ and Biden TJ. Molecular cloning and characterization of PKC iota, an atypical isoform of protein kinase $\mathrm{C}$ derived from insulinsecreting cells. J Biol Chem. 1993; 268:24296-24302.

16. Lamark T, Perander M, Outzen H, Kristiansen K, Overvatn A, Michaelsen E, Bjorkoy G and Johansen T. Interaction codes within the family of mammalian Phox and Bem1p domain-containing proteins. J Biol Chem. 2003; 278:3456834581 .

17. Gao L, Joberty G and Macara IG. Assembly of epithelial tight junctions is negatively regulated by Par6. Curr Biol. 2002; 12:221-225.

18. Regala RP, Weems C, Jamieson L, Copland JA, Thompson EA and Fields AP. Atypical protein kinase Ciota plays a critical role in human lung cancer cell growth and tumorigenicity. J Biol Chem. 2005; 280:31109-31115.

19. Regala RP, Thompson EA and Fields AP. Atypical protein kinase $\mathrm{C}$ iota expression and aurothiomalate sensitivity in human lung cancer cells. Cancer Res. 2008; 68:5888-5895.

20. Pia Rigobello M, Messori L, Marcon G, Agostina Cinellu M, Bragadin M, Folda A, Scutari G and Bindoli A. Gold complexes inhibit mitochondrial thioredoxin reductase: consequences on mitochondrial functions. J Inorg Biochem. 
2004; 98:1634-1641.

21. Murray NR, Kalari KR and Fields AP. Protein kinase Ciota expression and oncogenic signaling mechanisms in cancer. Journal of Cellular Physiology. 2011; 226:879-887.

22. Regala RP, Weems C, Jamieson L, Khoor A, Edell ES, Lohse CM and Fields AP. Atypical protein kinase $\mathrm{C}$ iota is an oncogene in human non-small cell lung cancer. Cancer Res. 2005; 65:8905-8911.

23. Messori L and Marcon G. Gold complexes in the treatment of rheumatoid arthritis. Met Ions Biol Syst. 2004; 41:279304.

24. Becker K, Gromer S, Schirmer RH and Muller S. Thioredoxin reductase as a pathophysiological factor and drug target. Eur J Biochem. 2000; 267:6118-6125.

25. Jeon KI, Jeong JY and Jue DM. Thiol-reactive metal compounds inhibit NF-kappa B activation by blocking I kappa B kinase. J Immunol. 2000; 164:5981-5989.

26. Kim NH, Lee MY, Park SJ, Choi JS, Oh MK and Kim IS. Auranofin blocks interleukin-6 signalling by inhibiting phosphorylation of JAK1 and STAT3. Immunology. 2007; 122:607-614.

27. Han S, Kim K, Kim H, Kwon J, Lee YH, Lee CK, Song Y, Lee SJ, Ha N and Kim K. Auranofin inhibits overproduction of pro-inflammatory cytokines, cyclooxygenase expression and PGE2 production in macrophages. Arch Pharm Res. 2008; 31:67-74

28. Kim NH, Park HJ, Oh MK and Kim IS. Antiproliferative effect of gold(I) compound auranofin through inhibition of STAT3 and telomerase activity in MDA-MB 231 human breast cancer cells. BMB Rep. 2013; 46:59-64.

29. Liu N, Li X, Huang H, Zhao C, Liao S, Yang C, Liu S, Song W, Lu X, Lan X, Chen X, Yi S, Xu L, Jiang L, Dong X, Zhou $\mathrm{P}$, et al. Clinically used antirheumatic agent auranofin is a proteasomal deubiquitinase inhibitor and inhibits tumor growth. Oncotarget. 2014; 5:5453-5471.

30. Fiskus W, Saba N, Shen M, Ghias M, Liu J, Gupta SD, Chauhan L, Rao R, Gunewardena S, Schorno K, Austin CP, Maddocks K, Byrd J, Melnick A, Huang P, Wiestner A, et al. Auranofin induces lethal oxidative and endoplasmic reticulum stress and exerts potent preclinical activity against chronic lymphocytic leukemia. Cancer Res. 2014; 74:25202532.

31. Marzano C, Gandin V, Folda A, Scutari G, Bindoli A and Rigobello MP. Inhibition of thioredoxin reductase by auranofin induces apoptosis in cisplatin-resistant human ovarian cancer cells. Free Radic Biol Med. 2007; 42:872881.

32. Tiekink ER. Gold derivatives for the treatment of cancer. Crit Rev Oncol Hematol. 2002; 42:225-248.

33. Mirabelli CK, Johnson RK, Hill DT, Faucette LF, Girard GR, Kuo GY, Sung CM and Crooke ST. Correlation of the in vitro cytotoxic and in vivo antitumor activities of gold(I) coordination complexes. J Med Chem. 1986; 29:218-223.

34. Mansfield AS, Fields AP, Jatoi A, Qi Y, Adjei AA,
Erlichman C and Molina JR. Phase I dose escalation study of the PKCiota inhibitor aurothiomalate for advanced nonsmall-cell lung cancer, ovarian cancer, and pancreatic cancer. Anticancer Drugs. 2013; 24:1079-1083. 